\title{
PROKLA-Redaktion
}

\section{Editorial: Arbeit, informell}

„Die kleinsten Kinder sind auf mancherlei Weise beschäftigt. Ein großer Teil derselben trägt Fische zum Verkauf...; andere sieht man sehr oft in der Gegend des Arsenals, oder wo sonst etwas gezimmert wird, wobei es Späne gibt, auch am Meere, welches Reiser und kleines Holz auswirft, beschäftigt, sogar die kleinsten Stückchen in Körbchen aufzulesen... Sie gehen nachher mit den Körbchen tiefer in die Stadt und setzen sich mit ihren kleinen Holzportionen gleichsam zu Markte... Andere Kinder tragen das Wasser der Schwefelquellen... Eine sehr große Anzahl von Menschen... beschäftigen sich, das Kehricht auf Eseln aus der Stadt zu bringen. Das nächste Feld... ist nur ein Küchengarten, und es ist eine Freude, $\mathrm{zu}$ sehen, welche unsägliche Menge von Küchengewächsen alle Markttage hereingeschafft wird und wie die Industrie der Menschen sogleich die überflüssigen, von den Köchen verworfenen Teile wieder in die Felder bringt, um den Zirkel der Vegetation zu beschleunigen... Ich würde zu weit aus meinem Wege gehen, wenn ich hier von der mannigfachen Krämerei sprechen wollte...; allein ich muß doch hier von den Herumträgern sprechen... Einige gehen herum mit Fäßchen Eiswasser, Gläsern und Zitronen...; andere tragen Körbe allerlei Backwerks, Näscherei. Zitronen und anderes Obst umher... Wie diese Art Herumträger geschäftig sind, so gibt es noch eine Menge kleiner Krämer, welche gleichfalls herumgehen und, ohne viele Umstände, auf einem Brett, in einem Schachteldeckel ihre Kleinigkeiten oder auf Plätzen, geradezu auf flacher Erde, ihren Kram ausbieten... Ich weiß wohl, daß dies viel zu allgemein gesagt ist und daß die Charakterzüge jeder Klasse nur erst nach einer genauern Bekanntschaft und Beobachtung rein gezogen werden können, allein im ganzen würde man doch, glaube ich, auf diese Resultate treffen..."

So beschreibt Johann Wolfgang von Goethe den ,,informellen Sektor“ Neapels in seinen Aufzeichnungen vom 28. Mai 1787 während der ,italienischen Reise“. Er ist soziologisch vorsichtig und macht die Aussagen von ,genauerer Beobachtung“ abhängig, meint aber doch Plausibilität für seine Darstellung beanspruchen zu können. Tatsächlich fallen die Beschreibungen der Lumpensammlerinnen in Cuiabá in Brasilien oder der Möbeltischler auf den Straßen Dakars oder von Verkäufern indigener Kunst in Mexiko-City ganz ähnlich aus. Heute bezeichnen wir diese Arbeiten als „,informell“. Es drängt sich daher der Eindruck auf, es handle sich dabei um eine ziemlich alte Geschichte. Auch die Beschreibungen vorindustrieller Arbeit von Marx oder in modernen wirtschaftshistorischen Studien ergeben ein ähnliches wie das von Goethe vom Ne- 
apel des ausgehenden 18. Jahrhunderts gezeichnete Bild. Nur sind die Farben weniger freundlich und die Schattenseiten der Tätigkeiten von Kindern, kleinen Händler und Handlangern und ihre Bedeutung für die ,ursprüngliche kapitalistische Akkumulation" werden deutlicher herausgestellt (vgl. zur Geschichte von Informalität die Beiträge von Bettina Musiolek und Dorothea Schmidt).

Wenn wir uns am Ende des 20. Jahrhunderts mit jenen Arbeiten befassen, die als informell gelten, haben wir zunächst das Seltsame des Begriffs von „Informalität“ oder „Informalisierung“ $\mathrm{zu}$ diskutieren. Informell ist eine $\mathrm{Be}$ stimmung der Abgrenzung zu dem, was als formell gilt. Und formell ist Arbeit dann, wenn sie bestimmten gesellschaftlichen Normen entspricht. Goethe wäre diese Unterscheidung fremd gewesen. Doch nach mehr als 200 Jahren Geschichte der Industriearbeit, die zugleich die Geschichte der Herausbildung und historischen Veränderung eines „Normalarbeitsverhältnisses“ “ ist, ist dessen vielfältig beschriebene Auflösung, ohne daß sich bislang eine neue „Normalität“ bzw. „Formalität“ der Arbeit ergeben hätte, gleichzeitig die Entstehung einer Welt der nicht-formellen, also informellen Arbeit. Und mehr noch: Die Normen der Arbeit, also diejenigen, die die Arbeitsbedingungen, die Arbeitszeiten, die Entlohnung, die geforderten Leistungen oder die soziale Sicherung regulierten und der Arbeit kulturelle Geltung verschafften, werden nicht mehr allein im nationalen Raum gesetzt, sondern in der globalen Konkurrenz ,aufgeherrscht“. Globale „,benchmarks“ müssen eingehalten werden, und diese bestimmen die Normen der Arbeit mehr als die Bedingungen, die in Tarifverhandlungen gesetzt werden.

Ein Resultat jedenfalls ist die tendenzielle Auflösung rigider Formen der Regulation von Lohn und Leistung und deren weitgehende Flexibilisierung. Die Regulation wird also „formlos“ und die Arbeit in diesem Prozeß ,informell“. Das reicht von der illusionären Autonomie der Scheinselbständigkeit über prekäre und nicht geschützte Arbeitsverhältnisse bis zur Steuern vermeidenden Schwarzarbeit. Die Zahl von Menschen, die unter diesen Arbeitsverhältnissen ,informell“ arbeiten, ist bereits hoch und in der Tendenz (in den westeuropäischen Ländern) zunehmend. Sie bewegt sich zwischen weniger als $10 \%$ der Erwerbspersonen in den nordischen und über $40 \%$ in mediterranen Ländern. Informelle Arbeit ist eine Antwort auf die Aussichtslosigkeit vieler Menschen, eine formelle, gesellschaftlich gesicherte Arbeit gemäß den etablierten Regeln des Sozialstaats zu finden. Die positive Seite besteht in der Entwicklung neuer, ,informeller Formen“, um die negative Seite, nämlich den Ausschluß von den Zugangsmöglichkeiten $\mathrm{zu}$ den gesellschaftlichen Gratifikationen zu vermeiden. Der Zugang ist in einer kapitalistischen Geldgesellschaft nur mit dem Medium des Geldes möglich. Da das Geld knapp ist, kann es in der Regel (also solange einer kein Geldvermögensbesitzer ist) nur durch Arbeit erworben werden. Wenn formelle Arbeit ebenfalls „knapp“ ist, eröffnet ,informelle“ Beschäftigung den Zugang zu den gesellschaftlichen Gratifikationen.

Mit dieser Überlegung gerät aber nur ein verschwindend kleiner Teil des globalen ,informellen Sektors“ ins Blickfeld. Erstens ist für die Gesellschaften der sogenannten „Dritten Welt“ in Af- 
rika, Lateinamerika und Asien festzuhalten, daß die nicht förmliche und nicht-normale Arbeit gesellschaftliche Normalität und Überlebensstrategie ist (vgl. dazu den Beitrag von Reinhart Kößler und Gerhard Hauck). Auch in den Transformationsökonomien Russlands scheint sich eher eine ,informelle Regulation" durchzusetzen, als eine an den Standards von OECD-Ländern orientierte marktwirtschaftliche Normalität (siehe dazu den Beitrag von Lydia Heller und Sabine Nuss).

Bis zu neun Zehnteln der Arbeitskräfte sind in der sogenannten 3 . Welt im informellen Sektor tätig: in der städtischen und ländlichen Kleinproduktion, im Straßenhandel, im Schmuggel. Damit sind wir zweitens an einer anderen Grenzlinie der Informalität, nicht an jener zum formellen, sondern an jener zur Illegalität und Kriminalität angelangt. Der Arbeitsprozeß oder/und das Arbeitsprodukt sind in manchen Bereichen nicht nur jenseits der gesellschaftlichen „weichen“, sondern jenseits der ,harten" legalen Normen. Handel als solcher ist legal, aber Handel mit Drogen, Waffen, Kindern und Frauen ist kriminell. Prostitution kann legal sein, aber bestimmte Formen sind es nicht. Migration kann legal und illegal sein. Die Arbeit, die Arbeitsbedingungen und die Entlohnung der MigrantInnen werden sehr verschieden sein, je nachdem ob sie legal oder illegal eingereist sind. Die „Deformalisierung“ der Arbeit eröffnet ganz neue Möglichkeiten der Ausbeutung, da sich die informellen Arbeitskräfte auf kein Gesetz berufen können, das sie schützt. Angesichts dessen stellt sich gerade für Afrika als dem am wenigsten ,entwickelten“ Kontinent die Frage, inwieweit diese Deformalisierungsprozesse überhaupt noch Raum für endogene Entwicklungen lassen (vgl. dazu den Aufsatz von Robert Kappel).

Drittens lagert der formelle Sektor zu einem Teil auf informeller Arbeit auf. Da informelle Arbeit häufig ,billiger“ $\mathrm{zu}$ haben ist als formelle Arbeit, ist es rentabel, Vorprodukte von Anbietern zu beziehen, die billige Arbeitskräfte beschäftigen. Honorige Konzerne aus den Industrieländern machen sich die Billigangebote der Zulieferer in Ländern zu Nutze, in denen förmliche Regeln für Arbeit und Leistung, für Arbeitsschutz und Interessenvertretung nicht oder unzureichend existieren. Daher werden Fußbälle in Pakistan genäht, Textilien in den Sonderwirtschaftszonen Chinas, in Bangla Desh oder in Bulgarien für europäische Bekleidungsketten gefertigt oder Turnschuhe in Vietnam oder Haiti geklebt. Die Informalität wird also von hoch-formalisierten Unternehmen, die für ihre eigenen Arbeitskräfte zumeist sehr förmliche Regeln in Tarifverträgen festgelegt haben, genutzt, um entweder eine Spaltung der in einer Produktionskette (von dem Rohprodukt Leder bis zum fertigen Fußball im Schaufenster) engagierten Arbeitskräfte zum Zwecke höherer Rentabilität aufrechtzuerhalten oder um so Druck auf die formell und daher sozial geschützten Beschäftigten auszuüben (vgl. dazu den Beitrag von Bettina Musiolek).

Viertens sind von den Prozessen der Informalisierung die Geschlechterverhältnisse betroffen, also Frauen anders als Männer (siehe dazu Aufsatz von Christa Wichterich). Die vielfach beschriebene Ungleichheit der Geschlechter in der ,formellen“ Arbeitswelt hochentwickelter Gesellschaften - dies trotz aller Gleichheitsrhetorik - setzt sich unter Bedingungen der Informalisierung fort, und zwar verstärkt. Im Fall von Prosti- 
tution, Kinder- und Frauenhandel, also modernen Formen der Sklaverei, ist die Betroffenheit deprimierend offensichtlich. Auch in den sweat shops von $\mathrm{Zu}-$ lieferern für die ,modernen“ Unternehmen der Industrieländer sind die Arbeitsverhältnisse von Frauen häufig weniger „förmlich“ als die von Männern, wenn zur „Form“ der Arbeit nicht nur disziplinierende Pflichten, sondern auch einklagbare Rechte gezählt werden. Darüber hinaus tragen Frauen unter Bedingungen der Armut sehr häufig die Verantwortung für die familiäre Subsistenz, für die funktionierende Haushaltsökonomie, ohne die Männer noch nicht einmal ihrer informellen Tätigkeit nachgehen könnten. So lagert nicht nur der formelle Sektor auf dem informellen Sektor auf, sondern der informelle Sektor in mancher Hinsicht auch auf der Subsistenz- und Haushaltsökonomie. Sie bietet eine geringe und immer prekäre Sicherheit gegen die Unsicherheiten, die in Markt- und Geldgesellschaften unvermeidlich sind und in langen Auseinandersetzungen in den Industrieländern durch Systeme sozialer Sicherung erträglich gestaltet werden konnten. Die soziale Sicherung wird überall reduziert, in weniger entwickelten Gesellschaften wird sie schon abgeschrieben und ideologisch im neoliberalen Diskurs verunglimpft, bevor sie eingeführt worden wäre und bevor die Menschen mit formellen Systemen der Regulation von Arbeit und der Sicherung von Lebensperspektiven hätten Erfahrungen machen können.

Fünftens bedeutet „Informalität“ keineswegs Regellosigkeit und Unsicherheit (was sowohl in dem Beitrag von Kössler/Hauck als auch in dem von Heller/Nuss deutlich wird). Nur finden die Regeln der ,modernen Industriege- sellschaft" mit ihrer Arbeit in formalisierten Verhältnissen keine Anwendung. Sie machen Arbeit zu teuer, zu rigide und verlangen innerhalb eines ökonomischen Systems auch in anderer Hinsicht Anpassungen, die die Wettbewerbsfähigkeit im globalen, aber auch regionalen, nationalen Raum unterminieren. Informalisierung ist also eine schlichte Strategie der Kostensenkung. Doch muß man sich davor hüten, das funktionalistische Argument zu überstrapazieren. Denn es gibt Regeln der Informalität, die weit komplexer sind, als nur die Kostensenkung zu betreiben; sie stellen einen gewissen Grad an sozialer Sicherheit und Verläßlichkeit dar. Die Regeln der Omerta der sizilianischen Mafia sind mehr als bloß funktionelle Erfordernisse des Selbstschutzes kriminineller Banden. Häufig gelten eher Reziprozitätsbeziehungen als solche der Äquivalenz, da Geld in manchen Sektoren eine eher mindere Rolle spielt. Ethnische Ökonomien im angelsächsischen oder südostasiatischen Raum sind nicht nur funktionelle Ökonomien, sondern den Regeln familiärer Sukzession und Autorität verpflichtet. Diese Andeutungen verweisen auf ein komplexes Forschungsprogramm: Welche Regeln regulieren informelle Arbeit, wenn denn die formellen Regeln unterminiert werden oder gar nicht erst etabliert werden können? Welche Rolle spielen dabei globale Standards, die eine gemeinsame Globalität vorspiegeln, die aber in der lokalen Realität von Gesellschaften und den ,informellen Formen“ ihrer Arbeitswelten gar nicht wiedergefunden werden kann?

Sechstens ist die Frage aufzuwerfen, welche Konsequenzen die Informalisierung der Arbeit für das Geld besitzt. Denn in kapitalistischen Gesellschaften 
erfolgt ja die Vergesellschaftung doppelt: durch Arbeit und durch Geld. Das Geld ratifiziert sozusagen die Gesellschaftlichkeit des Produkts der Arbeit; der Produzent gelangt ans Geld, mit dem er - oder sie - das Lebens bestreiten bzw. eine neue Runde der Produktion und Akkumulation einleiten kann. Geld verselbständigt sich auch im Kredit gegenüber der Arbeit, so daß es scheinen mag, als ob das Geld für diejenigen, die über Geldvermögen verfügen können, arbeite. Ergreift die Informalisierung auch das Geld? Dies ist zu vermuten, da Geld für informelle Arbeiten anders zirkuliert als Geld für formelle Arbeit. Diesen Sachverhalt machen sich Ökonomen zunutze, um die Größe des Schwarzmarkts zu messen. Unter der Annahme, daß für Schwarzarbeit nur Bargeld fließt, weil sie sonst zu leicht „,auffliegen“ könnte, läßt sich am Bargeldumlauf und seinen Veränderungen die Dynamik der ,Schattenwirtschaft" indizieren. Geld, das mit illegalen Aktivitäten verdient worden ist, muß „gewaschen“ werden - ein neues Aktivitätsfeld für einen informell-illegalen Sektor, nicht selten organisiert von formellen Institutionen des internationlen Kreditwesens. Geld kann auch aus der Zirkulation verschwinden und daher einem in manchen Fällen sehr umfangreichen Handel auf Gegenseitigkeit, Naturalientausch etc. Platz machen. Dies ist in Rußland nach der Finanzkrise von 1998 geschehen und dies ist in Afrika in großem Stil der Fall.

Siebentens strahlt die Informalisierung von Arbeit und Geld auf die politische Regulation aus. Informalität ist keineswegs Regellosigkeit, wurde gesagt. Doch entsteht die Regulierung der Informalität nicht selten an den förmlichen politischen Regeln, Normen, Insti- tutionen vorbei und manchmal gegen sie. Dies ist besonders eklatant bei kriminellen Aktivitäten, die sich über das Gesetz hinwegsetzen und in vielen Fällen trotzdem nichts zu befürchten haben, weil die staatliche Sanktionsgewalt unzureichend ist. Mit anderen Worten: In bestimmten Fällen kann Informalisierung bedeuten, daß die staatliche Kompetenz der Regelsetzung unterminiert und aufgehoben wird. Dies kann bis zum Zerfall von Staatswesen führen, auch wenn der Anlaß dafür immer ein anderer ist, wie im ehemaligen Jugoslawien. Die Informalisierung, manchmal Kriminalisierung nicht nur der Arbeit sondern des gesellschaftlichen Lebens haben zur Folge, daß ein neues Staatswesen nicht entsteht oder aber als Protektorat etabliert wird.

Seit Goethes Zeiten hat sich also nicht wenig in der Welt der Arbeit verändert. Wenn wir heute aus Neapel berichten würden, könnten wir immer noch auf die Fischverkäufer in Santa Lucia weisen, auf die Händler mit Limonade und Zuckerwatte in Mergellina, die Fahrradboten, die durch den Verkehr der Via Partenope flitzen. Aber damit hätten wir einen kleinen Sektor der Informalität im Blickfeld, nicht den großen Zigarettenschmuggel zwischen Nordafrika und Süditalien, den Industriezweig von Entführungen und Erpressungen, die organisierte Prostitution von mehr oder weniger freiwilligen Migrantinnen aus Afrika, Asien, Osteuropa, die Drogen, die aus Albanien und Montenegro unter dem NATO-Schirm eingeführt werden, die Camorra, die immer wieder nach der Macht in Stadt und Staat zu greifen versucht. Neapel ist Neapel, aber irgendwie auch eine der vielen Städte in globalisierter Welt, wo die Arbeit so ähnlich, informell, organisiert ist. 